\title{
An Inertial Coupled Marine Power Generator for Small Boats
}

\author{
S. M. Sharkh, M. Hendijanizadeh, M. Moshrefi-Torbati, M. Russell \\ School of Engineering Sciences \\ University of Southampton \\ E-mail: suleiman@soton.ac.uk, m.hendijanizadeh@soton.ac.uk,m.m.torbati@soton.ac.uk
}

\begin{abstract}
This paper proposes a device to harvest energy from the vertical motion of small boats and yachts. The device comprises a sprung mass coupled to an electrical generator through a ball screw. The mathematical equations describing the dynamics of the system are derived. The equations are used to determine the optimum device parameters, namely its mass, spring constant, ball screw lead, within practical constraints. Simulation results are presented to determine the maximum power that can be generated and the optimum load resistance as a function of boat vibration frequency.
\end{abstract}

Index Terms—Energy harvesting, Small boat, Vibration

Nomenclature

$\begin{array}{ll}m & \text { Mass } \\ x(t) & \text { Displacement of the mass } \\ k & \text { Spring constant } \\ B & \text { Damping factor of system } \\ l & \text { Ball screw lead or pitch } \\ \tau_{m} & \text { Damping torque } \\ \omega & \text { Generator rotational speed } \\ J & \text { Moment inertia of the ball screw and } \\ \tau_{G} & \text { generator } \\ k_{t} & \text { Generator torque } \\ i & \text { Generator torque constant } \\ k_{e} & \text { Generator current } \\ Z_{T} & \text { Generator EMF constant } \\ R_{L} & \text { Total impedance of load and generator } \\ v_{e m f} & \text { Load resistance } \\ Z_{0} & \text { Generator emf } \\ Y_{0} & \text { Amplitude of the relative displacement } \\ R_{G} & \text { of the mass } \\ L_{G} & \text { Amplitude of the displacement of the } \\ & \text { Goat } \\ & \text { Generator resistance } \\ & \end{array}$

\section{INTRODUCTION}

Cruising and racing yachts (overall length 10 to 30 meters) are increasingly reliant on electrical power for lighting, navigation equipment and for automatic steering systems, in the case of single handed sailing. Power requirement is typically in the 10 to 50 watt range and a 12 volt lead acid storage battery is commonly used. For most short range cruising conditions the battery is kept charged by the main engine. However, this is not allowed during racing and many cruising yachtsman would prefer to avoid using the main engine solely for battery charging.

Wind generators, towed impeller generators and solar cells are the most commonly used alternative power sources on small boats but they all have their own disadvantages. On relatively large racing yachts the wind and towed impeller devices tend to interfere with the rigging, and solar panels cannot provide enough power particularly in northern European waters. However, boat vertical motion is an existing source of energy and there exists the possibility of extracting energy from this renewable source of energy.

Harvesting energy from vibrating and moving structures, including those used in wave energy generators, has been the subject of significant research in recent years. Electrostatic and piezoelectric transducers are commonly utilized in miniature energy harvesting devices $[1,2]$. Electromagnetic energy convertors are typically used in larger devices. In most devices, the relative displacement between a relatively large inertia and the moving structure is exploited to drive a generator. In some inertial systems such as pendulum [3] or gyroscope [4] the energy conversion is based on relative rotation. In other devices, including the one described in this paper, relative linear motion is exploited. Direct drive linear generator have been used for energy conversion in these devices [5]. However, in applications where the frequency of vibrations, and hence the relative speed are low, a direct drive generator can be quite large and expensive relative to the amount of power produces, i.e. the power density of the generator will be very low. This is due to the fact that the size of an electric generator is proportional to its torque and accordingly the power density is proportional to its speed. To overcome this problem, some gearing is typically employed. Matsuoka et al. [6] for example used a ball screw to convert the linear displacement of a pressure plate and a floating body, in a wave energy harvesting device, to the rotational motion to 
drive an electric generator. Agamloh et al. [7] utilized the same method to convert the linear displacement of a moored buoy to rotational motion to drive a permanent magnet generator. Although there are some published papers applying this method to harvest energy from wave, most of them are related to either land based or tethered large scale power generation systems. However, in many applications with small power requirement the energy harvesting system should be portable. Brown et al. [8] reported their attempt to design a small wave energy generator for an autonomous underwater glider. They required an output power of $15 \mathrm{~W}$ for a maximum duration of 200 minutes. To produce this amount of energy they suggested a system in which a mass that oscillated around a center point set by a couple of springs. The system was designed to operate in a non-resonant mode. The paper however does not include information about the generator parameters.

This paper proposes an alternative small energy harvesting device that can be used to extract energy from the vertical motion of a small boat. It may also be used for small scale wave energy harvesting. The paper derives the dynamic equations of the device, which are used to determine its optimum parameters. Simulation results are presented to illustrate the performance of the device under different conditions of load and boat motion frequencies.

\section{SySTEM DESCRIPTION AND MODELING}

Fig. 1 shows a schematic diagram of proposed device. It comprises a sprung mass coupled to an electrical generator using a ball screw. The boat vertical motion will cause the mass to vibrate relative to boat. The equation of motion of the mass is:

$m \frac{d^{2} x(t)}{d t^{2}}=-k(x(t)-y(t))-B \frac{d(x(t)-y(t))}{d t}$

The damping factor $B$ is related to the power produced by the electrical generator and the friction forces in the system. Let the relative displacement of the system $x(t)-y(t)=z(t)$ hence the damping term in Equation (1) can be written as:

$B \frac{d(x(t)-y(t))}{d t}=B \frac{d z(t)}{d t}=f_{b}=\frac{2 \pi}{l} \tau_{m}$

where $l$ is the ball screw lead and the torque $\tau_{m}$ acting on the ball screw is:

$\tau_{m}=J \frac{d \omega(t)}{d t}+B_{m} \omega+\tau_{G}$

The electrical torque $\tau_{G}$ is given by:

$\tau_{G}=k_{t} i=k_{t} \frac{v_{e m f}}{Z_{T}}=\frac{k_{t} k_{e} \omega}{Z_{T}}$

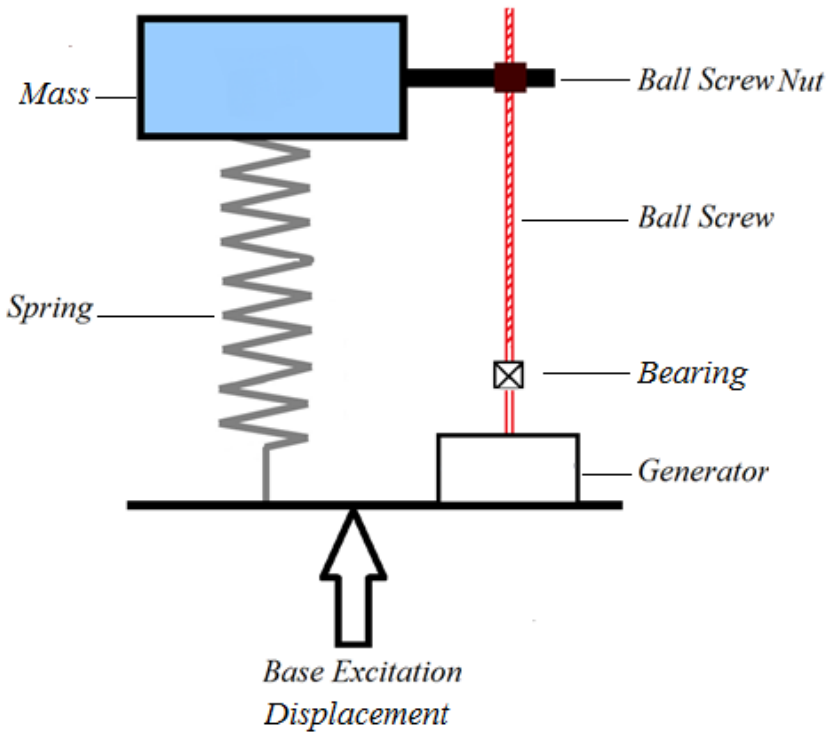

Fig. 1 Schematic diagram of system

The relationship between the linear speed of the mass and rotational speed of the ball screw and generator is given by:

$\omega=\frac{2 \pi}{l}\left(\frac{d z(t)}{d t}\right)$

Hence the Equation (2) can be arranged as follow:

$$
f_{b}=\frac{2 \pi}{l}\left(J \frac{2 \pi}{l} \frac{d^{2} z(t)}{d t^{2}}+B_{m} \frac{2 \pi}{l} \frac{d z(t)}{d t}+\frac{k_{t} k_{e}}{Z_{T}} \frac{2 \pi}{l} \frac{d z(t)}{d t}\right)
$$

And Equation (1) can be written as:

$$
\begin{aligned}
& (m+A) \frac{d^{2} x(t)}{d t^{2}}+D \frac{d x(t)}{d t}+k x(t)=A \frac{d^{2} y(t)}{d t^{2}}+D \frac{d y(t)}{d t} \\
& +k y(t)
\end{aligned}
$$

Where the reflected mass of the generator and the ball screw $A$ and the damping coefficient $D$ are defined as:

$A=J\left(\frac{2 \pi}{l}\right)^{2}$ and $D=\left(B_{m}+\frac{k_{t} k_{e}}{Z_{T}}\right)\left(\frac{2 \pi}{l}\right)^{2}$

And if the relative displacement $z(t)$ is of interest we can rewrite (7) as :

$(m+A) \frac{d^{2} z(t)}{d t^{2}}+D \frac{d z(t)}{d t}+K z(t)=-m \frac{d^{2} y(t)}{d t^{2}}$

If we assume a sinusoidal external displacement $y=Y_{0} \sin \omega t$ the amplitude of relative displacement $Z_{0}$ can be shown to be given by:

$$
\left|\frac{Z_{0}}{Y_{0}}\right|=\frac{m \omega^{2}}{\sqrt{\left(K-(m+A) \omega^{2}\right)^{2}+(D \omega)^{2}}}
$$




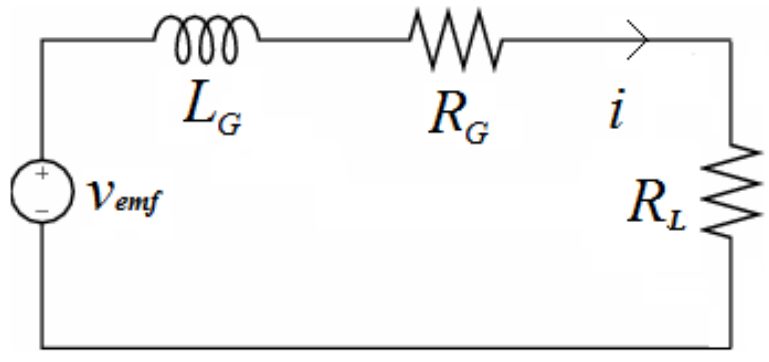

Fig. 2 Equivalent circuit of the DC generator and load

Based on the equivalent circuit of the DC generator and load shown in Fig. 2, the power supplied to the load can be related to the relative displacement using the previous equations:

$$
P=R_{L} i^{2}=R_{L}\left(\frac{v_{e m f}}{Z_{T}}\right)^{2}=\frac{R_{L}}{Z_{T}{ }^{2}} k_{e}{ }^{2}\left(\frac{2 \pi}{l} \frac{d z(t)}{d t}\right)^{2}
$$

Assuming sinusoidal boat displacement the maximum supplied to the load can be shown to be given by:

$$
\begin{aligned}
& P_{\max }=\frac{R_{L}}{Z_{T}{ }^{2}} k_{e}^{2}\left(\frac{2 \pi}{l}\right)^{2} \omega^{2} Y_{0}^{2} . \\
& \left(\frac{\left(m \omega^{2}\right)^{2}}{\left(K-\left(m+J\left(\frac{2 \pi}{l}\right)^{2}\right) \omega^{2}\right)^{2}+\left(\left(B_{m}+\frac{k_{e} k_{t}}{Z_{T}}\right)\left(\frac{2 \pi}{l}\right)^{2} \omega\right)^{2}}\right)
\end{aligned}
$$

\section{ANALYSIS AND SIMULATION RESUlts}

Equations (10) and (12) can be used to determine the optimal parameters of the system for a given excitation frequency. Fourier analyses of unpublished collected boat vertical acceleration during sailing of a small boat in the Solent and the English Channel indicated that dominate frequency of excitation is between 0.4 to $0.6 \mathrm{~Hz}$. For preliminary calculations we assume the frequency of excitation of boat to be $0.5 \mathrm{~Hz}$ with displacement amplitude of 1 meter.

Fig. 3 shows the ration of the amplitude of the relative displacement of the mass to the amplitude of the displacement of the boat as a function of the spring constant $k$, the generator and ball screw reflected mass $A$ and the damping coefficient $D$ for a given value of mass, and assuming an excitation frequency of $0.5 \mathrm{~Hz}$. As it can be seen by using a softer spring, larger relative displacement can be achieved, but we need a generator with a lower moment of inertia. Also, in each case the moment of inertia of the generator needs to be within a restricted range.

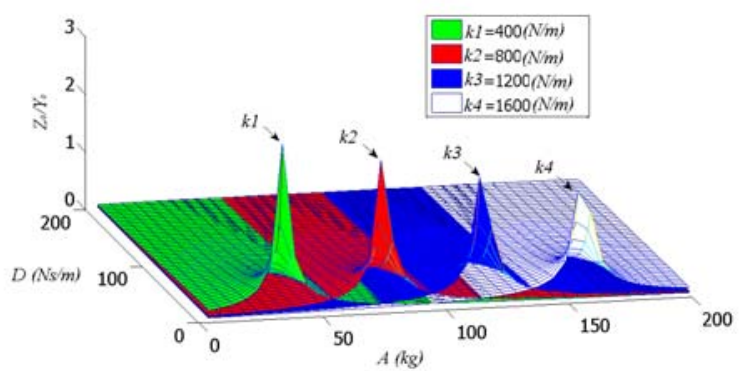

Fig. 3 Effect of the reflected generator and ball screw mass $A$, the damping coefficient $D$ and spring constant $k$ on the relative displacement of the mass of $(m=5 \mathrm{~kg}$ and $f=0.5 \mathrm{~Hz})$

Fig. 4 shows the effect of changing mass, generator and ball screw variables on relative displacement while the spring coefficient is constant. It is clear from the figure that a higher relative displacement is achieved when the mass is increased and the reflected mass of the ball screw and generator are reduced. Also, the damping coefficient $D$ can play an important role in controlling the relative displacement; however, we should not forget that from Equation (8), the damping coefficient has two terms. One term is related to the mechanical friction and losses in the ball screw and generator and this is proportional to the harvested energy. Another point that can be seen in Fig. 4 is relative displacement can be very high approaching nearly $20 \mathrm{~m}$ for typical boat displacements of $1 \mathrm{~m}$, which is not practical.

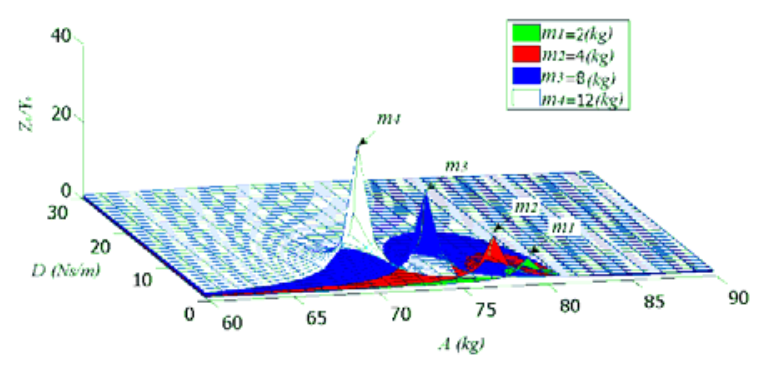

Fig. 4 Effect of $A, D$ and $m$ on relative displacement for a spring constant of $800 \mathrm{~N} / \mathrm{m}$ at a frequency of $0.5 \mathrm{~Hz}$

A design optimization study was carried out to determine the system parameters for a given brushless PM generator, which is capable of operating at high speed. The parameters of the generator were as shown in Table 1. A Matlab program was used to find the local peaks of generated power correspond to optimum combinations of mass, screw lead, spring constant and load resistance in the range is shown in Table. 2. The results show that a peak power of $470 \mathrm{~W}$ can be generated using the largest mass of $8 \mathrm{~kg}$. However, the corresponding relative displacement will be about $3.5 \mathrm{~m}$, which is not practical. 


\begin{tabular}{|c|l|}
\hline Parameters & \multicolumn{1}{|c|}{ Value } \\
\hline Rotor Inertia & 126 g.cm \\
\hline Torque Constant & $36 \mathrm{mN} . \mathrm{m} / \mathrm{A}$ \\
\hline Terminal Resistance & $0.45 \Omega$ \\
\hline Terminal Inductance & $0.71 \mathrm{mH}$ \\
\hline Mechanical Damping & $2.4 .10^{-4} \mathrm{mN} . \mathrm{m} / \mathrm{rpm}$ \\
\hline \multicolumn{2}{|c|}{ Table 1 - Generator parameters }
\end{tabular}

\begin{tabular}{|c|c|c|c|}
\hline Variable & Start & End & Increment(unit) \\
\hline$K$ & 200 & 1200 & 100 \\
\hline$l$ & 0.001 & 0.01 & 0.01 \\
\hline$m$ & 1 & 8 & 1 \\
\hline$R_{L}$ & 0 & 10 & 400 \\
\hline \multicolumn{3}{|c}{ Table 2 - Range of changing Parameters } \\
\hline
\end{tabular}

Limiting the amplitude of the relative displacement to $30 \mathrm{~cm}$, the optimum combination of parameters was found to be as follows: $k=400 \mathrm{~N} / \mathrm{m}, R_{L}=40 \Omega, l=0.004 \mathrm{~m}$ and $m=8 \mathrm{~kg}$. As expected, the largest mass generates the maximum power [9].

The above parameters were selected assuming that the excitation frequency is $0.5 \mathrm{~Hz}$, at which the system will resonate. In practice, the frequency will change depending on the speed of the boat, and the amplitude and wavelength of the waves [10]. To maximize the power output of the device at other excitation frequencies, the load resistance can be changed to shift the damped resonance frequency. Fig.5 shows the maximum power that can be harvested for a range of frequencies from $0.05 \mathrm{~Hz}$ to $1 \mathrm{~Hz}$. The load resistance was changed to achieve maximum power, such that the amplitude of the relative displacement does not exceed $30 \mathrm{~cm}$. This figure indicates that by increasing the frequency of excitation of the boat the maximum power generated will be increased, as expected.

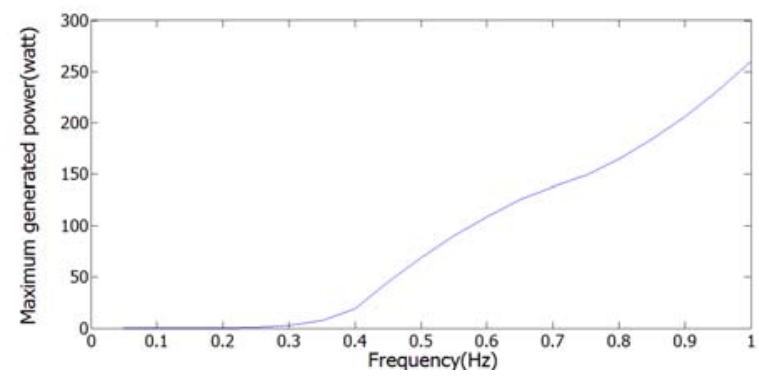

Fig. 5 Maximum produced power for different base excitation frequencies assuming a boat displacement amplitude of $1 \mathrm{~m}$

Fig. 6 shows the optimum load resistance versus excitation frequency for a device optimized to operate at a nominal frequency of $0.5 \mathrm{~Hz}$. This curve may be used as part of a maximum power tracking controller.

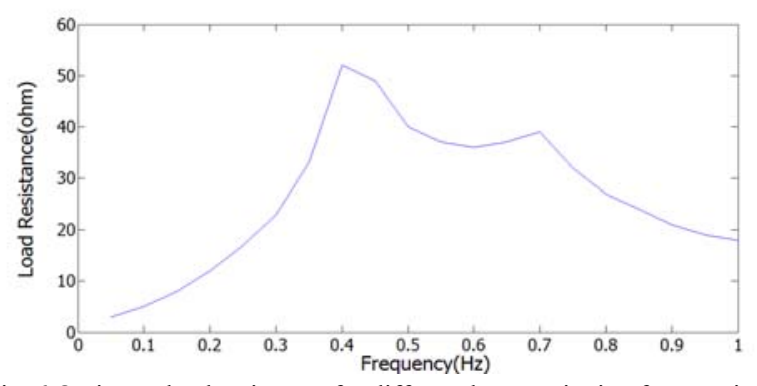

Fig. 6 Optimum load resistance for different base excitation frequencies to harvest the maximum power

\section{CONCLUSIONS}

The proposed device with a mass $m=8 \mathrm{~kg}$ (and a total mass of about $12 \mathrm{~kg}$ ) is estimated to produce about $75 \mathrm{~W}$ for a typical small boat motion with an amplitude of $1 \mathrm{~m}$ and a frequency of $0.5 \mathrm{~Hz}$. The parameters of the device need to be selected carefully to ensure resonance and limit the amplitude of oscillations of the mass to be within practical limits. It is also important to adjust the load resistance to ensure maximum power tracking.

\section{REFERENCES}

[1] M. Pereyma, "Overview of the Modern State of the Vibration Energy Harvesting Devices," Proc. Int. Conf on Perspective Technologies and Methods in MEMS Design, Lviv-Polyana, Ukraine, PP. 107-112, 2007.

[2] G. D. Pasquale, E. Brusa, A. Soma, "Capacitive Vibration Energy Harvesting with Resonance Tuning," Proc. of the Symposium on Design, Test, Integration \& Packaging of MEMS/MOEMS, Rome, Italy, pp. 280-285, 2009.

[3] M. Ruellan, H. Ben Ahmed, B. Multon, C. Josset, A. Babarit, A. Clement, "Design Methodology for a SEAREV Wave Energy Converter," IEEE Transactions on Energy Conversion, Vo. 25, No. 3, pp. 760-767, 2010.

[4] W. D. Jones, "Wringing watts from waves", IEEE Spectrum, Vol.42, No. 4, pp.14, 2005.

[5] R. Waters, M. Stalberg, O. Danielsson, O. Svensson, S. Gustafsson, E. Stromstedt, M. Eriksson, J. Sundberg, M. Leijon, , "Experimental results from sea trials of an offshore wave energy system," Applied Physics Letters, Vol. 90, No.3, pp. 034105 - 034105-3, 2007.

[6] T. Matsouka, K. Omata, H. Kanda, K. Tachi, "A study of wave energy conversion systems using ball screws - Comparison of output characteristics of the fixed type and the floating type", Proc. Int. Conf. on Offshore and Polar Engineering, Kitakyushu, Japan, pp. 581-585, 2002.

[7] E. B. Agamloh, A.K. Wallace, A. V. Jouanne, "A novel direct-drive ocean wave energy extraction concept with contact-less force transmission systems," Renewable Energy, Vol.33, pp. 520-529, 2008.

[8] P. Brown, D. Hardisty, T. C. A. Molteno, "Wave-powered small-scale generation systems for ocean exploration," Proc. Int. Conf. Oceans-Asia Pacific, Singapore, pp.1-6, 2006.

[9] C. B. Williams, R. B. Yates, "Analysis of a micro-electric generator for Microsystems," Sensors and Actuators, Vol. 52, pp. 8-11, 1996.

[10] P. Manganelli, Experimental investigation of dynamic loads on offshore racing yachts, $\mathrm{PhD}$ Thesis, School of engineering science, University of Southampton, Southampton, UK, 2006. 